Carolina Sales Vieira

Mariana Kefalás de Oliveira Gomes²

PaOla CÂNDIDO Rodrigues ${ }^{3}$

André Pera Marques Pinto ${ }^{4}$

Rosana Maria dos ReIS ${ }^{5}$

RuI Alberto Ferrian ${ }^{5}$

Marcos Felipe Silva de Sá ${ }^{5}$

Artigos originais

Palavras-chaves:

Síndrome do ovário policístico/fisiopatologia

Resistência à insulina

llhotas pancreáticas Insulina/secreção

Diabetes mellitus

Obesidade

Keywords:

Polycystic ovary

syndrome/physiopathology

Insulin resistance

Islets of Langerhans

Insulin/secretion

Diabetes mellitus

Obesity

Marcos Felipe Silva de Sá

Endereco: Campus Universitório

Departamento de Ginecologia e Obstetíícia da Faculdade de Medicina de Ribeirão Preto - FMRP/USP

Avenida Bandeirantes, 3900 (EP 14049-900 - Ribeirĩo Preto/SP

Fone: (16) 3602-2804 - Fax: (16) 3633-9633

E-mail: marcosfelipe@fmrp.usp.br

Recebido

03/04/2007

Aceito com modificacooes 12/02/2007

\section{Avaliação da função das células $\beta$ pancreáticas através do modelo matemático de HOMA em portadoras de síndrome dos ovários policísticos: comparação entre obesas e não-obesas}

\author{
B-cell function evaluation in patients with polycystic ovary syndrome \\ using HOMA model: a comparison between obeses e nonobeses
}

\section{Resumo}

OBJETIVO: avaliar o efeito da obesidade sobre a função das células $\beta$ pancreáticas de pacientes portadoras de síndrome dos ovários policísticos (SOP). MÉTODOS: estudo transversal no qual foram avaliadas 82 pacientes portadoras de SOP, selecionadas de forma consecutiva, no momento do diagnóstico de SOP. Pacientes com índice de massa corporal (IMC) maior ou igual a $30 \mathrm{~kg} / \mathrm{m}^{2}$ foram consideradas SOP obesas $(\mathrm{n}=31)$. Valores de índice de massa corporal menores que este limite foram consideradas SOP não-obesas, o que correspondeu a 51 mulheres. Foram utilizadas a glicemia e a insulina de jejum para cálculo da função das células $\beta$ pancreáticas $(H O M A-\% \beta-C e l l)$ e da resistência à insulina (HOMA-IR e QUICKI) entre os grupos. Analisaram-se, também, variáveis secundárias como idade, idade da menarca, níveis séricos hormonais (testosterona, prolactina, $\mathrm{LH}$ e FSH) e de colesterol total, triglicerídeos, HDL colesterol e LDL-colesterol. RESULTADOS: a idade da menarca das pacientes obesas com SOP (1 1,7 1,8 anos) foi menor que as não-obesas $(12,7 \pm 1,9)(p<0,05)$. As SOP obesas tiveram $L H$ inferior $(7,9 \pm 5,0 \mathrm{mUl} / \mathrm{mL})$ ao valor encontrado nas não-obesas $(10,6 \pm 6,0 \mathrm{mUl} / \mathrm{mL})(\mathrm{p}<0,05)$. Ambos os grupos apresentaram a média de $\mathrm{HDL}$ colesterol inferior a $50 \mathrm{mg} / \mathrm{dL}$. As pacientes obesas apresentaram insulina basal $(32,5 \pm 25,2 \mathrm{mUl} / \mathrm{mL})$ e glicemia de jejum $(115,9 \pm 40,7 \mathrm{mg} / \mathrm{dL})$ mais elevadas que as não-obesas $(8,8 \pm 6,6 \mathrm{mUI} / \mathrm{mL}$ e $90,2 \pm 8,9 \mathrm{mg} / \mathrm{dL}$, respectivamente) ( $\mathrm{p}<0,01)$. No grupo SOP obesas, a freqüência de resistência à insulina foi de 93 versus $25 \%$ no grupo SOP não-obesas $(p<0,01)$. Foi verificada hiperfunção das células $\beta$ do pâncreas em $86 \%$ das obesas com SOP contra $41 \%$ das não-obesas portadoras de SOP $(p<0,0001)$. CONCLUSÕES: as pacientes com SOP obesas apresentaram freqüência mais alta de resistência à insulina e hiperfunção de células $\beta$ do pâncreas quando comparadas com pacientes SOP não-obesas.

\section{Abstract}

PURPOSE: to evaluate the effect of obesity on $\beta$-cell function in patients with polycystic ovary syndrome (PCOS). METHODS: this cross-section study evaluated 82 patients with PCOS selected consecutively, at the moment of the diagnosis. We compared 31 PCOS obese women $\left(B M I \geq 30 \mathrm{~kg} / \mathrm{m}^{2}\right)$ to 51 age-matched PCOS nonobese patients $\left(\mathrm{BMl}<30 \mathrm{~kg} / \mathrm{m}^{2}\right)$. Using fasting glucose and insulin levels, homeostatic model assessment values for insulin resistance (HOMA-IR and QUICKI) and percent $\beta$-cell function (HOMA-\% $\beta$-cell) were calculated. As secondary variables, the age at PCOS diagnosis, age of menarche, hormonal levels (testosterone, prolactin, FSH and LH), total cholesterol, triglycerides, HDL cholesterol and LDL cholesterol were also analyzed. RESULTS: menarche was significantly earlier in obese PCOS patients (1 $1.7 \pm 1.8$ years) than in nonobese patients (12.67 1.86 years) ( $p<0.05)$. Obese patients presented lower $\mathrm{LH}$ levels $(7.9 \pm 5 \mathrm{mlU} / \mathrm{mL})$ than did nonobese patients $(10.6 \pm 6 \mathrm{mlU} / \mathrm{mL})(\mathrm{p}<0.05)$. Both groups presented mean $\mathrm{HDL}$ cholesterol levels below $50 \mathrm{mg} / \mathrm{dL}$. Obese patients presented significantly higher baseline insulin levels $(32.5 \pm 25.2 \mathrm{mlU} / \mathrm{mL})$ and fasting blood glucose levels (1 $15.9 \pm 40.7 \mathrm{mg} / \mathrm{dL})$ than did nonobese patients $(8.8 \pm 6.6 \mathrm{mlU} / \mathrm{mL}$ and $90.2 \pm 8.9 \mathrm{mg} / \mathrm{dL}$, respectively) ( $\mathrm{p}<0.01)$. Of the obese PCOS patients, $93 \%$ presented insulin resistance versus $25 \%$ of nonobese PCOS patients $(p<0.01)$. Eighty-six perecent of the obese women had hyperfunction of $\beta$-cell versus $41 \%$ of nonobese with PCOS ( $<<0.0001)$. CONCLUSIONS: obese PCOS patients presented higher prevalence of insulin resistance and hyperfunction of $\beta$-cell than did nonobese PCOS patients.

\section{Faculdade de Medicina de Ribeirão Preto da Universidade de São Paulo - USP - Ribeirão Preto (SP), Brasil.}

Médica Assistente do Departamento de Ginecologia e Obstetrícia da Faculdade de Medicina de Ribeirão Preto da Universidade de São Paulo - USP - Ribeirão Preto (SP), Brasil.

${ }_{2}$ Pós-graduanda do Departamento de Ginecologia e Obstetrícia da Faculdade de Medicina de Ribeirão Preto da Universidade de São Paulo - USP - Ribeirão Preto (SP), Brasil.

${ }^{3}$ Acadêmica do Curso de Medicina da Faculdade de Medicina de Ribeirão Preto da Universidade de São Paulo - USP - Ribeirão Preto (SP), Brasil; Bolsista de Iniciação Científica pelo Conselho Nacional de Desenvolvimento Científico e Tecnológico (CNPq).

${ }^{4}$ Acadêmico do Curso de Medicina da Faculdade de Medicina de Ribeirão Preto da Universidade de São Paulo - USP - Ribeirão Preto (SP), Brasil; Bolsista de Iniciação Científica pela Fundação de Amparo à Pesquisa do Estado de São Paulo (FAPESP).

5 Docentes do Setor de Reprodução Humana do Departamento de Ginecologia e Obstetrícia da Faculdade de Medicina de Ribeirão 


\section{Introdução}

A síndrome dos ovários policísticos (SOP) é a endocrinopatia mais comum no menacme, com prevalência de até $10 \%$ entre as mulheres em idade reprodutiva ${ }^{1,2}$. É caracterizada por hiperandrogenismo clínico e/ou laboratorial e anovulação crônica, excluindo-se outras causas de hiperandrogenismo ${ }^{3}$. Nos últimos anos, ampliou-se o conhecimento sobre outros distúrbios metabólicos associados à SOP, como a presença de hiperinsulinemia, resistência à insulina (RI), diabete mellitus (DM) tipo 2 de início precoce, dislipidemia e aumento de risco para doença cardiovascular ${ }^{4-6}$.

Aproximadamente metade das mulheres com SOP apresenta sobrepeso ou obesidade ${ }^{7}$. Acredita-se que a obesidade tenha um papel crucial no desenvolvimento e/ou manutenção da SOP e exerça grande influência nas alterações clínicas e metabólicas associadas à SOP, visto que uma pequena redução do peso (5\%) é capaz de melhorar o hiperandrogenismo e o padrão de anovulação presentes nas portadoras desta síndrome ${ }^{8}$.

Mais recentemente, a descoberta de alta prevalência de RI em portadoras de SOP, independentemente do peso, fez com que a RI passasse a ocupar uma posição de destaque na fisiopatologia da síndrome ${ }^{9}$. A RI chega a ser detectada em 50 a $90 \%$ das portadoras de SOP, com maior prevalência entre as pacientes com SOP e obesas, uma vez que a obesidade, por si só, é capaz de causar $\mathrm{RI}^{10,11}$. Foi proposto que, por vários mecanismos, a hiperinsulinemia leva ao aumento da produção de androgênios no ovario e na supra-renal, contribuindo para a anovulação ${ }^{11}$.

Além de RI, tem sido descrita em mulheres com SOP uma disfunção das células $\beta$ pancreáticas ${ }^{9,12,13}$, caracterizada, em alguns estudos, por aumento da secreção de insulina ${ }^{12,13}$, enquanto em outros, por redução da secreção dessa última ${ }^{14}$. Uma vez que a disfunção das células $\beta$ pancreáticas pode preceder a RI ou mesmo ser uma forma compensatória de manter a glicemia normal a despeito da $\mathrm{RI}^{9}$, é importante avaliar se há diferença na função das células $\beta$ pancreáticas de pacientes com SOP obesas e não-obesas, visto que a obesidade amplifica as características metabólicas adversas encontradas nas portadoras desta síndrome $e^{8,11}$.

Desta forma, este estudo tem como objetivo principal comparar a função das células $\beta$ pancreáticas de pacientes portadoras de SOP obesas e não-obesas.

\section{Métodos}

Foi conduzido um estudo transversal, no qual se compararam pacientes SOP obesas com não-obesas.

\section{Descrição da amostra}

Foram selecionadas todas as pacientes com diagnóstico de SOP realizado no período de janeiro de 2001 a dezembro de 2003, no Ambulatório de Ginecologia Endócrina do Hospital das Clínicas da Faculdade de Medicina de Ribeirão Preto da Universidade de São Paulo (FMRP/USP). A pesquisa foi aprovada pelo Comitê de Ética em Pesquisa do referido hospital.

Para diagnóstico de SOP, foram empregados os critérios do National Institute of Child Health and Human Development Consensus Conference ${ }^{3}$, que eram os vigentes na época (oligomenorréia ou amenorréia e hiperandrogenismo clínico e/ou laboratorial, sem outra causa aparente). O hiperandrogenismo clínico foi considerado presente quando o escore de Ferriman e Gallwey ${ }^{15}$ foi maior ou igual a 8. Laboratorialmente, considerou-se hiperandrogênica a paciente com níveis séricos de testosterona maiores que $100 \mathrm{ng} / \mathrm{dL}$ ou sulfato de deidroepiandrosterona maior que $300 \mu \mathrm{g} / \mathrm{dL}$. Todas as pacientes eram eutireóideas e normoprolactinêmicas.

O critério de inclusão foi o diagnóstico de SOP. Foram critérios de exclusão: diagnóstico de DM tipo 1 ou 2 e a presença de outras endocrinopatias que cursam com hiperandrogenismo clínico e/ou laboratorial (hiperprolactinemia, hiperplasia congênita da suprarenal formas clássica e não-clássica, tireoidopatias, tumores produtores de androgênio e síndrome/doença de Cushing).

As pacientes foram divididas em dois grupos de acordo com o índice de massa corporal (IMC). Pacientes com IMC maior ou igual a $30 \mathrm{~kg} / \mathrm{m}^{2}$ foram consideradas SOP obesas $(n=34)$. Valores de IMC menores que este limite foram considerados para definição de SOP nãoobesas, o que correspondeu a 51 mulheres.

Das pacientes SOP obesas, 8,8\% (3/34) apresentaram duas glicemias maiores ou iguais a $126 \mathrm{mg} / \mathrm{dL}$, sendo dessa forma excluídas por apresentarem DM tipo 2. No grupo das portadoras de SOP não-obesas não foi diagnosticada nenhuma paciente portadora de DM. Dessa forma, a amostra foi composta por 82 pacientes, sendo 31 pacientes SOP obesas e 51 SOP não-obesas.

\section{Variáveis analisadas e métodos empregados}

Foram avaliadas as seguintes variáveis para caracterização da amostra: idade, idade da menarca, presença de infertilidade (incapacidade para concepção sem métodos de contracepção após um ano de tentativa, com relações sexuais freqüentes), presença de hirsutismo (escore de Ferriman e Gallwey ${ }^{15}$ maior ou igual a 8) e volume ovariano médio avaliado pela ultra-sonografia.

Os níveis séricos de hormônio luteinizante (LH), hormônio folículo-estimulante (FSH), prolactina (PRL), 
testosterona, insulina de jejum, glicemia de jejum e perfil lipídico foram avaliados no momento do diagnóstico da doença. O perfil lipídico compreendeu a dosagem sanguínea de colesterol total (CT), colesterol da lipoproteína de alta densidade (HDL colesterol), colesterol da lipoproteína de baixa densidade (LDL colesterol) e triglicerídeos (TG).

Para a detecção da RI, utilizaram-se os índices de QUICKI (quantitative insulin sensitivity check index) e de HOMA-IR (homeostasis model assessment insulin resistance). $\mathrm{O}$ índice de QUICKI foi calculado pelas fórmulas de QUICKI = 1/[log (insulina jejum) $+\log$ (glicemia jejum) $]$ e de HOMA-IR $=[$ (glicemia de jejum em mg/dLx0,05551) x insulina de jejum em $\mu \mathrm{U} / \mathrm{mL}] / 22,5$. Diagnosticou-se como portadora de $\mathrm{RI}$ a paciente com QUICKI $\leq 0,34^{10}$ ou HOMA $>3,9 \mathrm{~mol} \times \mu \mathrm{U} / \mathrm{L}^{2,13}$.

Nenhuma paciente estava em uso de medicamentos que potencialmente alteravam os níveis séricos hormonais, o lipidograma e a insulina no momento da coleta.

Para avaliação da função da célula $\beta$ foi usada a fórmula matemática de HOMA - porcentagem de função da célula $\beta$ (HOMA-\% $\beta$-cell $)=[20 x$ insulina de jejum em $\mu \mathrm{U} / \mathrm{mL}] /[$ (glicemia em $\mathrm{mg} / \mathrm{dL} \times 0,05551$ ) - 3,5]. Uma mulher saudável no menacme tem $100 \%$ de função da célula $\beta$ pancreática. É considerado padrão hipersecretor quando HOMA-\% $\beta$-cell $>00 \%{ }^{16}$.

Para dosagens séricas de FSH, LH e PRL foi utilizada a quimioluminescência, em aparelho Immulite ${ }^{\circledR}$ DPC - Med Lab (Diagnostic Products Corporation - DPC, Los Angeles, CA, USA). A testosterona foi dosada por radioimunoensaio empregando o cintilador Tri Carb 2100 TR - Packard $^{\circledR}$.

A glicose plasmática foi avaliada pelo método hexoquinase, utilizando-se o aparelho Cobas Miras. A insulina sérica foi dosada em duplicata, utilizando-se o método de radioimunoensaio da DPC (Diagnostic Products Corporation), com a técnica sólida. As medidas foram feitas em analisador automático ANSR. A dose mínima detectável para insulina foi de $1,0 \mu \mathrm{UI} / \mathrm{mL}$. Os coeficientes de variação intraensaio foram 4,2 e 3,9\% e interensaio foram 9,7 e $8,6 \%$, respectivamente para glicose e insulina.

O CT, TG e HDL colesterol foram dosados por métodos enzimáticos com kits Roche ${ }^{\circledR}$ (Roche Diagnostics, Basel, Switzerland) - Unimat 5. O LDL colesterol foi calculado a partir da fórmula de Friedewald: LDL colesterol $=\mathrm{CT}-($ HDL colesterol $+\mathrm{TG} / 5)$, uma vez que não havia, nas amostras, dosagem de TG superior a $400 \mathrm{mg} / \mathrm{dL}$.

As análises estatísticas foram realizadas com o auxílio do software GraphPad Prism 3.0 ${ }^{\circledR}$ (Graphpad
Software, San Diego, CA, USA). A comparação entre os grupos (portadoras de SOP obesas e não-obesas) das variáveis paramétricas com distribuição normal foi realizada com teste $t$ de Student não pareado bicaudal (idade, idade da menarca, peso, altura, volume ovariano médio, glicemia, HOMA-\% $\beta$-cell, níveis séricos de LH, FSH, testorona, PRL, insulina, CT, HDL colesterol, LDL colesterol e TG). Para o IMC, que não apresentou distribuição normal, utilizou-se teste de Mann-Whitney bicaudal. A normalidade das variáveis foi realizada pelo teste de Kolmogorov-Smirnov. Os dados qualitativos foram comparados com o teste exato de Fisher (porcentagens de pacientes inférteis, de pacientes hirsutas, de pacientes com hiperfunção das células $\beta$ pancreáticas e de pacientes com resistência à insulina). Consideraram-se significativas as diferenças em que $\mathrm{p}<0,05$.

\section{Resultados}

As pacientes SOP obesas e não-obesas foram comparáveis quanto à idade (Tabela 1). As SOP obesas tiveram idade da menarca menor $(11,7 \pm 1,8$ anos) que as não-obesas $(12,7 \pm 1,9$ anos) $(\mathrm{p}=0,02)$. Aproximadamente metade das pacientes de cada grupo era hirsuta $(44,1 \%$ das obesas versus $53,8 \%$ das não-obesas), sem diferença entre os grupos. Mais de $70 \%$ das pacientes com SOP obesas e não-obesas apresentavam infertilidade, dado semelhante entre os grupos. Nenhuma diferença estatística foi encontrada quanto ao volume ovariano médio $\left(10,2 \pm 5,7 \mathrm{~cm}^{3}\right.$ nas obesas versus $12,7 \pm 7,8 \mathrm{~cm}^{3}$ nas não-obesas), porém os valores ultrapassaram o limite superior da normalidade $\left(9,0 \mathrm{~cm}^{3}\right)$ em ambos os grupos (Tabela 1 ), demonstrando aumento ovariano nas portadoras de SOP, independentemente do peso.

Tabela 1 - Características clínicas das pacientes com síndrome dos ovários policísticos (SOP).

\begin{tabular}{l|c|c}
\hline & Obesas $(\mathrm{n}=31)$ & Não-obesas $(\mathrm{n}=51)$ \\
\hline Idade (anos) & $28,3 \pm 6,3$ & $24,9 \pm 6$ \\
Peso (kg) & $95,1 \pm 19,0^{\star}$ & $60,9 \pm 8,3$ \\
Alłura (m) & $1,6 \pm 0,1$ & $1,6 \pm 0,1$ \\
IMC (kg/ $\left.{ }^{2}\right)$ & $37,2 \pm 6,5^{\star \star}$ & $24,3 \pm 3,2$ \\
Menarca (anos) & $11,7 \pm 1,8^{\star}$ & $12,7 \pm 1,9$ \\
Infertilidade (\%) & 85,3 & 73,1 \\
Hirsutismo (\%) & 44,1 & 53,8 \\
Volume ovariano médio $\left(\mathrm{cm}^{3}\right)$ & $10,2 \pm 5,7$ & $12,7 \pm 7,8$ \\
\hline
\end{tabular}

Valores expressos em média \pm desvio padrão; $I M C=$ =índice de massa corporal.

${ }^{*} \mathrm{p}<0,05$ comparado com as pacientes SOP não-obesas, pelo teste $t$ de Student.

** $p<0,05$ comparado com as pacientes SOP não-obesas pelo teste de Mann-Whitney. 
Quanto ao perfil hormonal, as SOP obesas tiveram níveis séricos de $\mathrm{LH}$ inferiores $(7,9 \pm 5 \mathrm{mUI} / \mathrm{mL})$ ao valor encontrado entre as não-obesas $(10,6 \pm 6,0 \mathrm{mUI} / \mathrm{mL})$ $(\mathrm{p}=0,04)$. Porém, não houve diferença entre os grupos em relação ao FSH, PRL e testosterona (Tabela 2).

Com relação ao perfil lipídico, não houve diferença

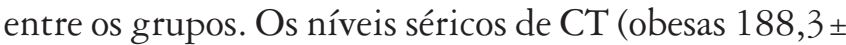

Tabela 2 - Características hormonais das pacientes com síndrome dos ovários policísticos (SOP).

\begin{tabular}{lcc}
\hline & Obesas $(\mathrm{n}=31)$ & Não-obesas $(\mathrm{n}=51)$ \\
\hline FSH (mUl/mL) & $4 \pm 1,7$ & $4,6 \pm 2,1$ \\
LH (mUl/mL) & $7,9 \pm 5^{*}$ & $10,6 \pm 6,0$ \\
PRL (ng/mL) & $9,7 \pm 4,37$ & $11,5 \pm 8,9$ \\
Testosterona (ng/dL) & $74,8 \pm 19,2$ & $83,1 \pm 30$ \\
\hline
\end{tabular}

Valores expressos em média \pm desvio padrão; FSH=hormônio folículo-estimulante; $\mathrm{LH}=$ hormônio luteinizante; $\mathrm{PRL}=$ prolactina .

${ }^{*} \mathrm{p}<0,05$ comparado com as pacientes SOP não-obesas pelo teste $t$ de Student.

Tabela 3 - Comparacão de variáveis bioquímicas entre pacientes com síndrome dos ovários policísticos (SOP) obesas e não-obesas.

\begin{tabular}{lcc}
\hline & Obesas $(\mathrm{n}=31)$ & Não-obesas $(\mathrm{n}=51)$ \\
\hline Glicemia $(\mathrm{mg} / \mathrm{dL})$ & $115,9 \pm 40,7^{\star}$ & $90,2 \pm 8,9$ \\
Insulina ( $\mathrm{pUl} / \mathrm{mL})$ & $32,5 \pm 25,2^{\star}$ & $8,8 \pm 6,6$ \\
HOMA-\% $\%$-cell & $270 \% \pm 149 \%{ }^{\star}$ & $91,5 \% \pm 58,2 \%$ \\
Colesterol total (mg/dL) & $188,3 \pm 41,0$ & $190,3 \pm 49,3$ \\
HDL colesterol (mg/dL) & $42,3 \pm 11,2$ & $45,4 \pm 12,1$ \\
LDL colesterol (mg/dL) & $114,7 \pm 34,8$ & $113,2 \pm 38,6$ \\
Triglicerídeos (mg/dL) & $123,3 \pm 41,1$ & $99,7 \pm 40,4$ \\
\hline
\end{tabular}

Valores expressos em média \pm desvio padrão; $\mathrm{HDL}$ =lipoproteína de alta densidade; LDL=lipoproteína de baixa densidade; HOMA-\% $\beta$-cell=porcentagem de função da célula $\beta$ pancreática pelo modelo de HOMA.

${ }^{*} p<0,05$ comparado com as pacientes SOP não-obesas pelo teste $t$ de Student.

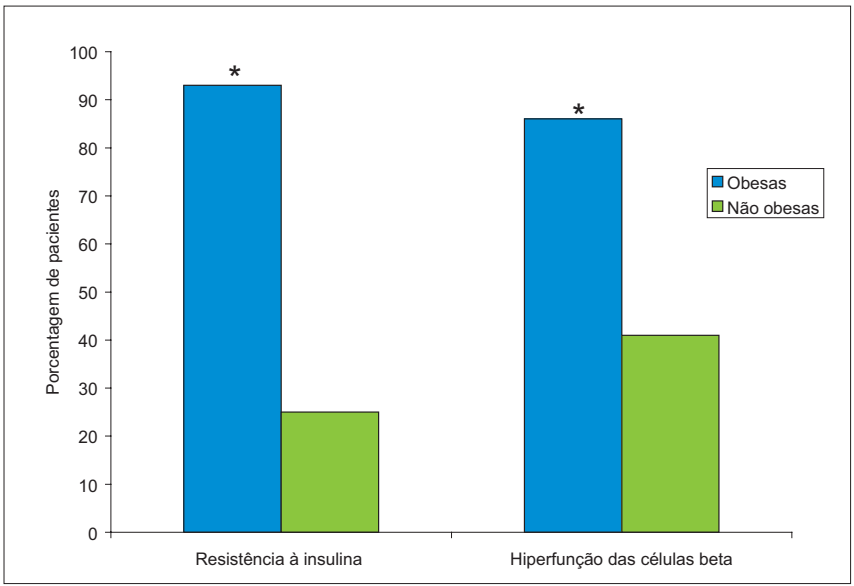

A resistência à insulina foi calculada pelos índices de HOMA e QUICKI. ${ }^{*} \mathrm{p}<0,05$ comparado com as pacientes SOP não-obesas pelo teste exato de Fisher.

Figura 1 - Freqüência de resistência à insulina e disfunção das células $\beta$ pancreáticas em pacientes com síndrome dos ovários policísticos (SOP): comparação entre obesas e não-obesas.
$41 \mathrm{mg} / \mathrm{dL}$ versus não-obesas 190,3 $\pm 49,3 \mathrm{mg} / \mathrm{dL}), \mathrm{TG}$ (obesas 123,3 $\pm 41,1 \mathrm{mg} / \mathrm{dL}$ versus não-obesas 99,75 \pm $40,45 \mathrm{mg} / \mathrm{dL}$ ), LDL colesterol (obesas 114,7 $\pm 34,8 \mathrm{mg} /$ $\mathrm{dL}$ versus não-obesas $113,2 \pm 38,6 \mathrm{mg} / \mathrm{dL}$ ) e de $\mathrm{HDL}$ C (obesas 42,3 $\pm 11,2 \mathrm{mg} / \mathrm{dL}$ versus não-obesas $45,4 \pm$ $12,1 \mathrm{mg} / \mathrm{dL}$ ) não apresentaram diferenças relacionadas à presença da obesidade (Tabela 3).

As pacientes obesas apresentaram níveis séricos de insulina basal $(32,5 \pm 25,2 \mu \mathrm{UI} / \mathrm{mL})$ e glicemia de jejum $(115,9 \pm 40,7 \mathrm{mg} / \mathrm{dL})$ significativamente mais elevados que as não-obesas $(8,8 \pm 6,6 \mu \mathrm{UI} / \mathrm{mL}$ e $90,2 \pm 8,9 \mathrm{mg} / \mathrm{dL}$ (Tabela 3 ). Em relação à $\mathrm{RI}$, no grupo das SOP obesas, $93 \%$ apresentaram RI (índice de QUICKI $\leq 0,34$ ) versus $25 \%$ no grupo SOP não-obesas $(\mathrm{p}<0,01)$ conforme Figura 1. O índice de HOMA-IR mostrou a mesma freqüência de RI entre os grupos do que o índice de QUICKI.

Por último, 86\% (27/31) das pacientes obesas com SOP possuem padrão hipersecretor de insulina (HOMA-\% $\beta$-cell $>100 \%)$ versus $41 \%(21 / 51)$ das não-obesas $(\mathrm{p}<0,0001)$ conforme Figura 1 , configurando aumento da função das células $\beta$ pancreáticas preferencialmente entre as portadoras de SOP obesas. A média de porcentagem da função das células $\beta$ pancreáticas em SOP obesas foi $270 \pm 149 \%$ (média \pm desvio padrão) versus $91,5 \pm 58,2 \%$ (média \pm desvio padrão) entre as não-obesas $(\mathrm{p}=0,001)$ (Tabela 3). Entre as não-obesas, $16 \%$ das pacientes $(8 / 51)$ já apresentavam padrão hipersecretor de insulina, mesmo na ausência de RI, o que não ocorreu com as portadoras SOP obesas, uma vez que todas as estas com hiperfunção das células $\beta$ pancreáticas também apresentavam RI.

\section{Discussão}

Neste estudo, o achado mais importante foi o fato de a obesidade promover um padrão hipersecretor das células $\beta$ pancreáticas. Das pacientes com SOP obesas, $86 \%$ apresentavam hiperfunção das células $\beta$ pancreáticas versus $25 \%$ das portadoras de SOP não-obesas.

A SOP por si só é capaz de alterar o padrão de secreção da insulina, independente do peso, conforme demonstrado previamente ${ }^{12}$. A maioria dos estudos, que utilizaram o mesmo método de avaliação da função das células $\beta$ pancreáticas (HOMA-\% $\beta$-cell), relatam um aumento da secreção de insulina em pacientes com SOP comparadas com controles saudáveis ${ }^{12,13,17}$. Uma hipótese para explicar a hiperfunção das células $\beta$ do pâncreas, em pacientes com SOP, é que esta seria uma forma compensatória de manter a glicemia normal, devido à $\mathrm{RI}$, freqüentemente observada nestas pacientes. Com a sobrecarga crônica destas células, estas apresentam risco 
de evoluir para exaustão de sua função, e as pacientes de desenvolver intolerância à glicose ou DM tipo $2^{9,17}$.

Outra possibilidade seria uma alteração genética nas células $\beta$ pancreáticas das pacientes com SOP, responsável pela hipersecreção de insulina nessas pacientes ${ }^{9,18}$. Confirmando essa última hipótese, em nosso estudo observamos que $16 \%$ das pacientes não-obesas com SOP apresentavam hiperfunção das células $\beta$, porém sem evidências de RI, o que não foi verificado entre as obesas com SOP.

Neste estudo, a obesidade associou-se a uma maior secreção pancreática de insulina. Este resultado foi consoante com os achados de outros estudos anteriores, que mostraram maior freqüência de padrão hipersecretor de insulina entre as portadoras de SOP obesas comparadas àquelas sem obesidade ${ }^{12,17}$. Outros autores demonstraram correlação positiva entre a função das células $\beta$ pancreáticas e obesidade ${ }^{13}$. Existem estudos que relataram redução da secreção de insulina entre obesas com SOP e aumento da secreção de insulina em pacientes magras com $\mathrm{SOP}^{19}$.

Estas diferenças, encontradas entre os diversos estudos, das comparações entre a função das células $\beta$ de pacientes com SOP obesas e magras podem se dever a uma porcentagem maior de pacientes com intolerância à glicose ou DM entre as obesas selecionadas nestes estudos, que demonstraram diminuição da secreção de insulina em obesas, uma vez que essas morbidades estão associadas à deterioração da função das células $\beta$ do pâncreas ${ }^{9}$. Em nosso estudo, excluímos as pacientes com SOP com DM da avaliação de função das células $\beta$ e de RI. A prevalência de DM tipo 2 antes da quarta década de vida, em nossa amostra de SOP obesas, foi de $8,8 \%$, resultado comparável à prevalência de $10 \%$ reportada em outro estudo 5 .

O método que usamos para avaliação da função das células $\beta$ pancreáticas é o modelo de HOMA, que usa glicemia e insulina plasmáticas de jejum para cálculo matemático da porcentagem de função das células $\beta$ do pâncreas ${ }^{20}$. As estimativas da função das células $\beta$ pancreáticas pelo modelo de HOMA apresentam boa correlação com o padrão-ouro (clamp glicêmico) para a avaliação da secreção de insulina pelas células $\beta$, com a vantagem de esse método de ser mais barato, exequível e rápido ${ }^{7,13}$.

Tanto mulheres com SOP magras quanto obesas apresentam evidências de redução à sensibilidade da insulina, sendo esta mais marcante quando a obesidade está presente ${ }^{8,13}$. A prevalência de RI entre as obesas, no presente estudo, foi de $93 \%$, contra $25 \%$ entre as não-obesas com SOP, avaliada pelo índice de QUICKI e HOMA-IR, o que não foi surpresa, uma vez que o índice de QUICKI é a transformação logarítmica do HOMA-IR ${ }^{20}$. A alta prevalência de RI em pacientes obesas com SOP (93\%), em nosso estudo, foi comparável à prevalência de RI encontrada por outros autores em pacientes italianas obesas com SOP $(95,3 \%)$, usando o mesmo método para diagnóstico de resistência à insulina ${ }^{10}$.

A natureza da presença da hiperinsulinemia e de RI em pacientes com SOP ainda não está completamente esclarecida. Foi descrito um defeito pós-ligação ao receptor de insulina na transdução de sinais que se manifesta como alteração da fosforilação do receptor de insulina e do substrato do receptor de insulina tipo 1 . Essa anormalidade permanece mesmo em células de cultura, o que é consistente com uma alteração intrínseca da SOP, provavelmente genética ${ }^{21}$.

Juntamente com o risco aumentado de DM, a prevalência de dislipidemia está aumentada nas mulheres com SOP, especialmente níveis mais elevados de TG e inferiores de HDL colesterol ${ }^{17,22}$. A obesidade é, freqüentemente, associada a um perfil lipídico mais aterogênico em pacientes com $\mathrm{SOP}^{7}$. No presente estudo, não se evidenciou alteração no perfil lipídico entre os grupos, resultado semelhante aos achados de outros autores ${ }^{23}$. No entanto, independente do peso, as pacientes com SOP, deste estudo, apresentaram níveis séricos de HDL colesterol inferiores a $50 \mathrm{mg} / \mathrm{dL}$, um dos fatores presentes na síndrome plurimetabólica ${ }^{24}$, comumente descrita entre as portadoras de $\mathrm{SOP}^{22}$. Já o CT, o LDL colesterol e TG de ambos os grupos mostraram valores normais na casuística estudada, contrariando os resultados de outro estudo que relatou o aumento dos níveis de LDL colesterol e CT, como as alterações lipídicas predominantes em portadoras de SOP, independentemente da presença de obesidade ${ }^{25}$. Essa diferença de achados de anormalidades lipídicas entre os estudos pode se dever a diferenças étnicas, genéticas e ao estilo de vida das pacientes recrutadas ${ }^{22}$.

Em conclusão, a RI, a hiperfunção das células $\beta$ pancreáticas e a hiperinsulinemia estão presentes em alta freqüência nas portadoras de SOP obesas e não-obesas, porém, a sua magnitude é maior nas pacientes com SOP obesas, como mostraram os resultados do presente estudo. Como existem pacientes não-obesas, nas quais a hiperfunção das células $\beta$ pancreáticas está presente mesmo na ausência de RI, é importante a avaliação das células $\beta$ em portadoras de SOP, pois outros fatores devem estar implicados no mecanismo da hiperinsulinemia.

O reconhecimento desses achados na nossa população envolve buscar modalidades terapêuticas para a SOP que visam reduzir a hiperinsulinemia e prevenir a falência das células $\beta$, especialmente entre as obesas, que apresentam amplificação das alterações do metabolismo glicídico encontradas na SOP. Além dos agentes sensibilizadores de insulina, medidas que tenham como objetivo a perda de peso são fundamentais para melhorar a sintomatologia e restaurar a função ovariana e metabólica nas pacientes obesas com SOP. 
1. Azziz R, Woods KS, Reyna R, Key TJ, Knochenhaver ES, Yildiz $B O$. The prevalence and features of the polycystic ovary syndrome in an unselected population. J Clin Endocrinol Metab. 2004; 89(6):2745-9.

2. Asuncion M, Calvo RM, San Millan JL, Sancho J, Avila S, EscobarMorreale HF. A prospective study of the prevalence of the polycystic ovary syndrome in unselected Caucasian women from Spain. J Clin Endocrinol Metab. 2000; 85(7):2434-8

3. Zawadski JK, Dunaif A. Diagnostic criteria for polycystic ovary syndrome: towards a rational approach. In: Dunaif A, Givens JR, Haseltine F, Merriam GR, editors. Polycystic ovary syndrome. Boston: Blackwell Scientific; 1992. p. 377-84.

4. Carmina E, Lobo RA. Polycystic ovary syndrome (PCOS): arguably the most common endocrinopathy is associated with significant morbidity in women. J Clin Endocrinol Metab. 1999; 84(6):1897-9.

5. Ehrmann DA, Barnes RB, Rosenfield RL, Cavaghan MK, Imperial J. Prevalence of impaired glucose tolerance and diabetes in women with polycystic ovary syndrome. Diabetes Care. 1999; 22(1):141-6.

6. Dokras A, Jagasia DH, Maifeld M, Sinkey CA, VanVoorhis BJ, Haynes WG. Obesity and insulin resistance but not hyperandrogenism mediates vascular dysfunction in women with polycystic ovary syndrome. Fertil Steril. 2006; 86(6):1702-9.

7. Gambineri A, Pelusi C, Vicennati V, Pagotto U, Pasquali R. Obesity and the polycystic ovary syndrome. Int J Obes Relat Metab Disord. 2002; 26(7):883-96

8. Barber TM, McCarthy MI, Wass JA, Franks S. Obesity and polycystic ovary syndrome. Clin Endocrinol (Oxf). 2006; 65(2):137-45

9. Goodarzi MO, Erickson S, Port SC, Jennrich RI, Korenman SG. $\beta$-cell function: a key pathological determinant in polycystic ovary syndrome. J Clin Endocrinol Metab. 2005; 90(1):310-5.

10. Carmina E, Lobo RA. Use of fasting blood to assess the prevalence of insulin resistance in women with polycystic ovary syndrome. Fertil Steril. 2004; 82(3):661-5.

11. Salehi M, Bravo-Vera R, Sheikh A, Gouller A, Poretsky L. Pathogenesis of polycystic ovary syndrome: what is the role of obesity? Metabolism. 2004; 53(3):358-76.

12. Vrbíkova J, Bendlová B, Hill M, Vanková M, Vondra K, Stárka L. Insulin Sensitivity and $\beta$-cell function in women with polycystic ovary syndrome. Diabetes Care. 2002; 25(7):1217-22.

13. DeUgarte CM, Bartolucci AA, Azziz R. Prevalence of insulin resistance in polycystic ovary syndrome using the homeostasis model assessment. Fertil Steril. 2005; 83(5):1454-60.
14. Dunaif A, Finegood DT. $\beta$-cell dysfunction independent of obesity and glucose intolerance in the polycystic ovary syndrome. J Clin Endocrinol Metab. 1996; $81(3): 942-7$.

15. Ferriman D, Gallwey JD. Clinical assessment of body hair growth in women. J Clin Endocrinol Metab. 1961; 21:1440-7.

16. Matthews DR, Hosker JP, Rudenski AS, Naylor BA, Treacher DF, Turner RC. Homeostasis model assessment: insulin resistance and $\beta$-cell function from fasting plasma glucose and insulin concentrations in man. Diabetologia. 1985; 28(7):412-9.

17. Hahn S, Tan S, Elsenbruch S, Quadbeck B, Herrmann BL, Mann $\mathrm{K}$, et al. Clinical and biochemical characterization of women with polycystic ovary syndrome in North Rhine-Westphalia. Horm Metab Res. 2005; 37(7):438-44.

18. Colilla S, Cox NJ, Ehrmann DA. Heritability of insulin secretion and insulin action in women with polycystic ovary syndrome and their first degree relatives. J Clin Endocrinol Metab. 2001; 86(5):2027-31.

19. Arslanian SA, Lewy VD, Danadian K. Glucose intolerance in obese adolescents with polycystic ovary syndrome: roles of insulin resistance and $\beta$-cell dysfunction and risk of cardiovascular disease. J Clin Endocrinol Metab. 2001; 86(1):66-71.

20. Wallace TM, Levy JC, Matthews DR. Use and abuse of HOMA modeling. Diabetes Care. 2004; 27(6):1487-95.

21. Dunaif $A$. Insulin resistance in women with polycystic ovary syndrome. Fertil Steril. 2006; 86 Suppl 1:S13-4.

22. Carmina E, Napoli N, Longo RA, Rini GB, Lobo RA. Metabolic syndrome in polycystic ovary syndrome (PCOS): lower prevalence in southern Italy than in the USA and the influence of criteria for the diagnosis of PCOS. Eur J Endocrinol. 2006; 154(1):141-5.

23. Faloia E, Canibus P, Gatti C, Frezza F, Santangelo M, Garrapa $G G$, et al. Body composition, fat distribution and metabolic characteristics in lean and obese women with polycystic ovary syndrome. J Endocrinol Invest. 2004; 27(5):424-9.

24. Grundy SM, Cleeman II, Daniels SR, Donato KA, Eckel RH, Barry $A$, et al. Diagnosis and management of the metabolic syndrome: an American Heart Association/National Heart, Lung, and Blood Institute Scientific Statement. Circulation. 2005; $112(17): 2735-52$.

25. Legro RS, Kunselman AR, Dunaif A. Prevalence and predictors of dyslipidemia in women with polycystic ovary syndrome. Am J Med. 2001; $111(8): 607-13$. 